\title{
Genetic characterisation of small ruminant lentiviruses in sheep and goats from the Czech Republic
}

\author{
Pavel Barták ${ }^{1}$, Bronislav Šimek ${ }^{1}$, Petr Václavek ${ }^{1}$, Vladislav Čurn², Hana Plodková1, \\ Tomáš Tonka ${ }^{2}$, Barbora Farková ${ }^{2}$, Kateřina Vernerová2 ${ }^{2}$ Antonín Vejčík ${ }^{2}$ \\ ${ }^{1}$ State Veterinary Institute Jihlava, Jihlava, Czech Republic \\ ${ }^{2}$ University of South Bohemia, Faculty of Agriculture, Biotechnological Centre, \\ České Budějovice, Czech Republic \\ Received December 18, 2017 \\ Accepted April 3, 2018
}

\begin{abstract}
The aim of this study was to determine the prevalence of small ruminant lentivirus (SRLV) infections on sheep and goat farms which are exempt from state monitoring and carry molecular characterisation of strains circulating amongst these farms without SRLV eradication. A total number of 3,410 blood samples of sheep and goats from 21 herds were collected for the purpose of the project. The detected serological prevalence of maedi visna in sheep was $19.9 \%(556 / 2801)$ and the seroprevalence of caprine arthritis and encephalitis in goats was $14.1 \%(86 / 609)$. All positive animals were tested by the nested polymerase chain reaction (nPCR) method for the presence of provirus in the buffy-coats from EDTA-blood samples. Phylogenetic analysis of 93 SRLV strains identified the genotype in 77 sequences, where 60 of them were genotype A and 17 belonged to genotype B. Whereas all of the genotype B sequences were classified in subtype B2, the genotype A group of isolates showed higher variability and were related to subgenotypes A2 and A3. This study represents the first report of genetic characterisation of SRLV strains circulating in the territory of the Czech Republic.
\end{abstract}

Maedi visna, caprine arthritis, nested PCR, ELISA

Small ruminant lentiviruses (SRLVs) are widespread in most regions of the world and infections are associated with economic losses in small-ruminant production. The members of this SRLV group in the Retroviridae family, maedi-visna virus (MVV) and caprine arthritis-encephalitis virus (CAEV), infect sheep and goats (L'Homme et al. 2011). Small ruminant lentiviruses cause persistent infections which can induce systemic diseases affecting joints, mammary glands and respiratory and central nervous systems after long incubation periods (Narayan et al. 1983). Lentiviruses have in common their typical genomic organisation, the induction of slowly progressive diseases, a large spectrum of targeted organs and symptoms, and the ability to persist within their hosts despite a strong immunological response. Ingestion of infected colostrum and milk is the major transmission mode of SRLV within flocks (Blacklaws et al. 2004) but, at least in sheep, horizontal transmission may also play a prominent role (Narayan and Clements 1989). Common clinical signs caused by SRLV infections include neurological disorders, dyspnoea, emaciation, mastitis, and arthritis (Alvarez et al. 2006).

The genomic organisation of SRLVs is typical of lentiviruses: the proviral genome consists of two identical positive-sense single-stranded ribonucleic acid (RNA) subunits (8.4-9.2 kb) (Gifford 2012). The SRLV genome contains the structural genes gag, pol and $e n v$, in addition to the regulatory genes rev, vif, and tat. The gag and pol genes are relatively well conserved among SRLVs, which makes them ideal targets for Polymerase Chain Reaction (PCR) primer design (Pepin et al. 1998; Grego et al. 2007). The MVV prototypical strains such as strain K1514, EV-1, and SA-OMVV from sheep and the CAEV 
strain Cork-CAEV isolated from goat (L'Homme et al. 2011) were originally viewed as distinct viral species restricted to respective host animal species. As more SRLV sequences had become available for phylogenetic analyses, it became evident that SRLVs can cross the species barrier and can be transmitted from sheep to goats under favourable conditions (Shah et al. 2004b; Pisoni et al. 2005).

The classification of viral genotypes into groups and subtypes proposed in the last decade by Shah et al. (2004a) involving two long segments of the SRLV genome (gag-pol segment $1.8 \mathrm{~kb}$; and pol segment $1.2 \mathrm{~kb}$ ) is widely accepted at present. Accordingly, SRLV are classified into five genotype groups (A-E) which can vary between $25 \%$ and $37 \%$ in their nucleotide sequences. However, genotypes A, B and E may further be distributed into different subtypes, varying between $15 \%$ and $27 \%$ of their sequence. Group A has so far 15 recognised subtypes (A1-A15); group B has three subtypes (B1-B3); and group $\mathrm{E}$ has only two subtypes (E1 and E2). Genotype D has only been described in Swiss and Spanish sheep, and only regarding pol sequences. Rather, phylogenetic analysis on additional ( $\mathrm{gag}$ ) sequences of the same (group D) isolates, classify these sequences with genotype A, suggesting that genotype D is in fact genotype A, exhibiting divergence in the pol gene (Ramírez et al. 2013). The ocurrence of genotype $\mathrm{E}$ is described in local goat populations in the northwestern region of Italy and the island of Sardinia (Grego 2007; Reina 2010). The high genetic variability amongst SRLV and the corresponding phylogenetic relationships amongst sequences has been described in numerous studies in different countries (Ramírez et al. 2013). Although SRLV infection in small ruminants is widespread on all continents, little information is available on the genetic variants circulating in different geographic regions. Besides SRLV complete genomes available in the GenBank, partial sequences have been published in Brazil, Canada, Finland, France, Greece, Ireland, Japan, Netherlands, Poland, Russia, Slovenia, South Korea, Turkey (reviewed by Ramírez et al. 2013), Switzerland (Shah et al. 2004a), Italy (Grego et al. 2002), and Norway (Gjerset et al. 2007).

In the Czech Republic MVV/CAEV was firstly described in the early 1990s based on the serological diagnosis by detection of specific antibodies using commercial agar gel immunodiffusion test (AGID). First virus isolation and partial genome characterisation of the Czech ovine lentivirus isolate OPM was published by Celer et al. in 1997. Further six Czech ovine lentivirus isolates were characterised from snPCR products of amplification of partial gag gene sequences (Celer et al. 2000), which confirmed the ovine lentivirus genotype in the Czech Republic is closely related to the prototype Maedi-Visna strains K1514 (Iceland), EV1 (Scotland) and SA-OMVV (South Africa), recently grouped to the A1 subgroup. Nevertheless, clear phylogenetic differences can be found between the six viruses analysed and the first OPM isolate.

We tested a large number of sheep and goats throughout the Czech Republic within the framework of our research project aimed to control the lentiviral infections in small ruminants with the use of early detection methods of SRLVs. All samples were tested for the presence of SRLV serum antibodies using different ELISA tests (Enzyme-Linked ImmunoSorbent Assay). Selected samples from SRLV positive farms were further tested by PCR to amplify proviral sequences of sufficient length suitable for the genetic typing of SRLVs circulating in the country. The final goal of the project is also the genetic selection on the basis of markers of genetic resistance to infection (TMEM154 gene).

\section{Materials and Methods}

Animals and blood sampling

Animals at least 4 months after weaning, originating from 21 farms from 7 different regions of the Czech Republic (Karlovy Vary, Liberec, Olomouc, Pardubice, Pilsen, South Bohemian and South Moravian Region) were used for the study. A total of 3,410 blood samples of sheep and goats were collected for the purpose of the project, 
of which 2,801 were ovine samples from 16 herds and 609 caprine samples from 7 herds. Two farms were mixed flock with sheep and goats. Whole blood from sheep and goats at a volume of $5 \mathrm{ml}$ was drawn by venipuncture and collected into tubes with ethylenediaminetetraacetic acid (K EDTA).

\section{ELISA tests}

All samples were tested for SRLV antibodies by commercially available ELISA test (IDEXX MVV/CAEV p28 Ab Screening Test) and the findings were confirmed by additional ELISA assays IDEXX MVV/CAEV p28 Ab Verification Test (IDEXX, USA), IDEXX CAEV/MVV Total Ab Test (IDEXX, USA), Elitest MVV/CAEV (HYPHEN BioMed, France), ID Screen ${ }^{\circledR}$ MVV/CAEV Indirect (IDvet, France) and one agar gel immunodiffusion test AGID MAEDITEC (APHA Scientific, UK). Eradikit SRLV ELISA test (IN3 Diagnostics, Italy) based on a mix of gag and env peptides belonging to the genotypes A and B was used for serological genotyping.

\section{Buffy-coat preparation and white blood cell isolation}

White blood cells (WBC) were isolated from K3EDTA whole blood by centrifugation at $400 \times g$ for 20 min at room temperature (RT). After careful removal of plasma for serological testing, buffy coat layer was transferred by pipetting to clean $1.5 \mathrm{ml}$ tubes. Second centrifugation at $400 \times \mathrm{g}$ for $20 \mathrm{~min}$ was used for the residual plasma removal. Final cleaning of buffy coat was performed using the lysis solution $\left(\mathrm{NH}_{4} \mathrm{Cl}, \mathrm{NaHCO}_{3}\right.$, EDTA, final $\mathrm{pH}$ 7.3). Samples were centrifuged at $1,000 \times g$ for $5 \mathrm{~min}$ at RT and the supernatant was discarded. The obtained pelet was stored at $-80^{\circ} \mathrm{C}$.

\section{Deoxyribonucleic acid extraction}

Deoxyribonucleic acid (DNA) from WBC was extracted using a MagNA Pure LC Total NA Isolation kit on MagNA Pure robotic system (Roche). Prelysis step was performed using lysis solution (GuSCN, Tris- $\mathrm{HCl}$ (pH 6.4), EDTA (pH 8.0), Triton X-100) in the volume of $300 \mu 1$ and $(50 \mathrm{mg} / \mu \mathrm{l})$ in the volume of $3 \mu \mathrm{l}$ in a thermomixer $\left(56^{\circ} \mathrm{C}\right.$ for $\left.90 \mathrm{~min}\right)$. The Proteinase $\mathrm{K}$ enzyme was subsequently deactivated at $96{ }^{\circ} \mathrm{C}$ for $20 \mathrm{~min}$.

\section{Polymerase chain reaction}

Two-pair primers (Table 1) corresponding to the most highly conserved sequences in the gag gene from the targeted SRLV genomes were used for nested PCR (Grego et al. 2007). The primers GAG F1 and POL R1 were used for the first PCR step, where the consequent length of the amplification product was $0.8 \mathrm{~kb}$. The second set of primers GAG F2 and POL R2 was used for nested PCR step carried out in a $25 \mu 1$ reaction volume. The extracted DNA was amplified in a reaction mixture of the total volume of $25 \mu 1$ with the following composition: primers $(0.3 \mu \mathrm{M}$ for each), Deoxynucleotide (dNTPs) Solution Mix $(0.12 \mathrm{mM}), 1 \times$ PCR buffer complete with $\mathrm{MgCl}_{2}(0.12 \mathrm{mM})$, Fast Start Taq Polymerase (1 Unit). The temperature profile started with an initial denaturation step $\left(95^{\circ} \mathrm{C}\right.$ for $\left.10 \mathrm{~min}\right)$, followed by 35 cycles with denaturing $\left(95^{\circ} \mathrm{C}\right.$ for $\left.1 \mathrm{~min}\right)$, annealing $\left(55^{\circ} \mathrm{C}\right.$ for $\left.30 \mathrm{~s}\right)$ and elongation $\left(72^{\circ} \mathrm{C}\right.$ for $\left.1 \mathrm{~min}\right)$. Nested PCR was carried out under similar conditions as the first PCR, where $2 \mu 1$ of the first PCR product were transferred into the reaction mix. The temperature profile was the same as in the first PCR, except for the annealing step $\left(60^{\circ} \mathrm{C}\right.$ for $\left.30 \mathrm{~s}\right)$.

Table 1. Specific primers for the gag gene region designed according to Grego et al. (2007).

\begin{tabular}{llc}
\hline Primer & \multicolumn{1}{c}{ Sequence 5' $-3^{\prime}$} & $\begin{array}{c}\text { Length of PCR product } \\
\text { Length of nPCR } \\
\text { product }\end{array}$ \\
\hline GAG F1 & TGGTGARKCTAGMTAGAGACATGG & $1.3 \mathrm{~kb}$ \\
POL R1 & CATAGGRGGHGCGGACGGCASCA & \\
\hline GAG F2 & CAAACWGTRGCAATGCAGCATGG & $0.8 \mathrm{~kb}$ \\
POL R2 & GCGGACGGCASCACACG & \\
\hline
\end{tabular}

PCR: polymerase chain reaction; nPCR: nested polymerase chain reaction; kb: kilobyte

\section{Sequencing}

The specificity of the resulting PCR products can be verified using the sequence analysis and by comparing the obtained sequences with referential sequences available in the GenBank at the National Center for Biotechnology Information (NCBI). Polymerase chain reaction products with specific length were cut out from agarose gel and purified on columns (High Pure PCR Product Purification Kit [Roche, Germany] and Expin combo GP [GeneAll Biotechnology, Korea]). Purified DNA was sequenced by Sanger method with reamplification primers using dye terminator chemistry. The output sequence data were processed in the BioEdit software (Tom Hall, 
Ibis Biosciences, Carlsbad, CA, USA) and modified sequences were compared with sequences in the NCBI database via the BLAST (Basic Local Alignment Search Tool) algorithm. Based on this comparison, supposed incorporation was estimated of detected proviral sequences to the SRLV genotype groups and their subtypes.

Phylogenetic analysis

Obtained nucleotide sequences were analyzed, adjusted and preliminarily compared by Bio Edit software (Hall 1999). Multiple alignment of analysed sequences together with reference SRLV sequences was accomplished with Clustal W in MEGA 7 (Kumar et al. 2016). Phylogenetic and molecular evolutionary analyses were performed using the Neighbour Joining method and the Tamura-Nei model with gamma substitution in MEGA 7 (Nei and Kumar 2000) and all gaps and missing data were ignored. The statistical confidence of the topologies was assessed using 1000 bootstrap replicates.

Table 2. Results of serological testing, PCR and genetic typing on Czech farms.

\begin{tabular}{|c|c|c|c|c|c|c|c|c|}
\hline \multirow{2}{*}{ Farm } & \multirow{2}{*}{ Sample code } & \multirow{2}{*}{ Species } & \multirow{2}{*}{ Nr. of animals } & \multicolumn{2}{|c|}{ ELISA } & \multirow{2}{*}{ PCR positive } & \multicolumn{2}{|c|}{ Genotype } \\
\hline & & & & $\mathrm{Ab+}$ & $\mathrm{Ab}-$ & & gag gene & genotype ELISA \\
\hline A & Po & sheep & 243 & 211 & 32 & 29 & $\mathrm{~A} 2 / \mathrm{A} 3$ & NDet \\
\hline A & $\mathrm{Pk}$ & goat & 431 & 389 & 42 & 18 & $\mathrm{~A} 2 / \mathrm{A} 3$ & A \\
\hline B & $\mathrm{Br}$ & sheep & 150 & 126 & 24 & 11 & $\mathrm{~A} 2 / \mathrm{A} 3$ & A \\
\hline $\mathrm{C}$ & Sch & sheep & 230 & 210 & 20 & 14 & $\mathrm{~A} 2 / \mathrm{A} 3$ & A,B \\
\hline D & M & sheep & 200 & 103 & 97 & 70 & $\mathrm{~A} 2 / \mathrm{A} 3$ & A \\
\hline $\mathrm{E}$ & $\mathrm{Ab}$ & sheep & 300 & 91 & 209 & 139 & $\mathrm{~B} 2, \mathrm{~A} 2 / \mathrm{A} 3$ & B \\
\hline $\mathrm{F}$ & $\mathrm{Sl}$ & sheep & 113 & 74 & 39 & 23 & $\mathrm{~A} 2 / \mathrm{A} 3$ & NDet \\
\hline G & Zo & sheep & 59 & 45 & 14 & 14 & $\mathrm{~A} 2 / \mathrm{A} 3$ & A \\
\hline G & $\mathrm{Zk}$ & goat & 274 & 120 & 154 & 92 & $\mathrm{~A} 2 / \mathrm{A} 3$ & A \\
\hline total & & & 2000 & 1369 & 631 & 410 & & \\
\hline
\end{tabular}

ELISA: Enzyme-Linked ImmunoSorbent Assay; PCR: polymerase chain reaction

\section{Results}

A total number of 3,410 blood samples of sheep and goats from 21 herds were tested for presence of specific antibodies against MVV/CAE. Specific SRLV antibodies were detected in 642 animals on 7 farms out of 21 (33\%). The detected serological prevalence of MVV in sheep was $19.9 \%(556 / 2801)$ and the prevalence of CAE in goats was $14.1 \%(86 / 609)$. The proviral DNA was detected by PCR in 410 samples $(65 \%)$ out of 631 seropositive animals. A total of 287 suitable reamplification products were sequenced amongst the 410 PCR positive animals from 7 farms (Table 2). The length of the analysed sequences for the phylogenetic tree was adjusted to about $750 \mathrm{bp}$ (1002-1752 according to the location of the complete genome sequence of the reference strain M60609.1-Icelandic strain K1514).

Small ruminant lentivirus sequences were compared to reference strains from genotype groups A, B, C and E. Due to the high number of positive samples within infected farms, a total of 77 samples representative of each flock were randomly selected for further phylogenetic analysis. Amongst the 77 gag gene sequences, genotype A was identified in 60 of them, and 17 belonged to genotype B. A total of 40 representative sequences (5-8 for each farm) are presented in the phylogenetic tree ( 35 of them belonging to genotype A and 5 of them to genotype B) (Fig. 1). Whereas all the genotype B sequences clustered clearly in subtype B2, the genotype A group of isolates showed higher variability and were related to subgenotypes A2 and A3. Detailed comparison of partial nucleotide sequences from Farm E with reference strains A1, A2, and A3 (Fig. 2) revealed circulation of more than one subtype (A2/A3 and B2) in this flock.

Nucleotide diversity within each group was compared in group A (mean nucleotide diversity, $15.3 \%$; range, $0.1-22.5 \%$ ) and group $\mathrm{B}$ (mean, $2.4 \%$; range, $0.2-9.0 \%$ ). Intra- 


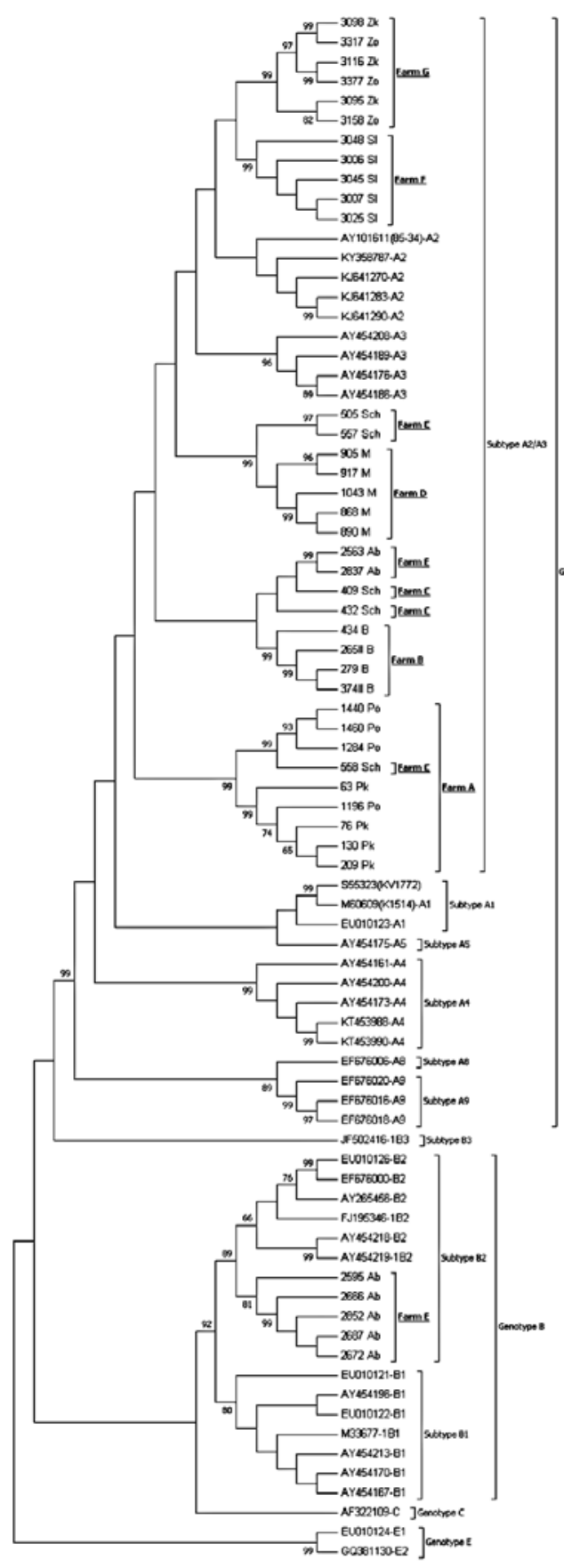

Fig. 1. Phylogenetic relationship of goat and sheep lentivirus nucleotide sequences of isolates from Czech flocks by the Neighbour joining method. farm heterogenity in group $\mathrm{A}$ : farm $\mathrm{A}$ (mean, 6.0\%; range, 1.0-18.4\%), farm B (mean, 11.4\%; range, 3.4-15.5\%), farm C (mean, 14.7\%; range, 2.4-21.0\%), farm $\mathrm{D}$ (mean, $4.3 \%$; range, $0.1-10.7 \%$ ), farm E (mean, 5.6\%; range, $0.4-8.4 \%$ ), farm $\mathrm{F}$ (mean, 2.4\%; range, $0.1-3.8 \%$ ), farm G (mean, 9.4\%; range, $0.3-15.2 \%)$ and group B: farm E (mean, 2.4\%; range, $0.2-9.0 \%$ ).

Results of serological examination of 90 serum samples by subunit ELISA on genotype $\mathrm{A}$ and $\mathrm{B}$ derived matrix protein (Eradikit SRLV ELISA test) confirmed the presence of genotypes detected by molecular genotyping on 6 out of 7 farms, while on one of them (farm C) a suspected additional presence of genotype $\mathrm{B}$ besides genotype A was revealed by sequence analysis. Otherwise, on farm E showing circulation of genotypes A and B, solely genotype $B$ was presented in all significant results $(33 / 55)$. Even particular samples tested positive for genotype A by PCR reacted in this ELISA obviously with a B subunit antigen.

\section{Discussion}

A 'gold standard' for the detection of SRLV infection has not existed up until now. Tools recommended for early detection consist of laboratory methods for detection of the humoral immune response (serology) and methods detecting the (pro-)viral nucleid acid. The proviral DNA was detected by PCR in 410 samples $(65 \%)$ out of 631 seropositive animals. This result corresponds to past studies (Celer et al. 1997; Shah et al. 2004a; De Andrés et al. 2005) where the sensitivity of various PCR approaches related to different ELISA tests vary from 60 to $95 \%$. Polymerase chain reaction tests tend to be less sensitive than many ELISA tests regarding the identification of infected individuals by laboratory testing (De Andrés et al. 2005). Polymerase chain reaction appears to 


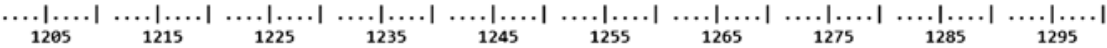

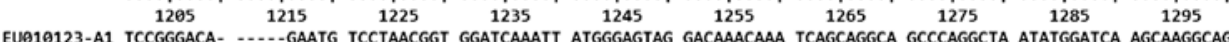
KY358787-A2 GCCCGGGCC. -....-AGGTG TCCTTACAGT GGATCAAATC ATGGGAGTAG GACAAACAAA TCATCAGGCA TCACAAGCTA ATATGGATCA AGCAAGGCAA 2512 Abn-A GCCAGGGCC- -....-AAATG TCCTTACAGT GGATCAAATT ATGGGAGTAG GACAAACAAA TCAGCAGGCA TCACAAGCCA ATATGGATCA GGCAAGGCAG AY454176-A3 GCCAGGGCC. -...-AAATG TCCTTACAGT GGATCAAATC ATGGGAGTAG GACAAACAAA TCAGCAAGCG GCGCAAGCCA ATATGGATCA AGCAAGACAA EUఅ10120-B1 ACCACCTCCA GCAGGAGGAG CACTAACAGT GGATCAAATT ATGGGGGTAG GACAAACGAA TCAAGCAGCA GCACAAGCTA ACATGGATCA GGCAAGACAA 2424 Abn-B2 ACCACCTCCG GCAGGAGGAG GACTAACCGT GGATCAAATT ATGGGAGTAG GACAAACAAA TCAAGCAGCT GCTCAAGCTA ACATGGATCA AGCAAGACAA AY454218-B2 ACCACCTCCG GCAGGAGGAG GATTAACAGT GGATCAAATT ATGGGAGTAG GACAAACAAA TCAGGCAGCT GCACAAGCTA ACATGGATCA AGCAAGGCAA

\begin{tabular}{|c|c|c|c|c|c|c|c|c|c|c|}
\hline & 13 & $\begin{array}{r}\because 3 \\
13\end{array}$ & 13 & 13 & 13 & 13 & 13 & $13 / 5$ & 1 & \\
\hline$-A 1$ & ATATGTITGC & AATGGGTAAT & AACAGCATTA & AGATCAGTAA & GGCATATGTC & GCATAGACCA & GGAAACCCTA & TGCTAGTCAA & GCAG & trat \\
\hline /358787-A2 & TATGCTTGC & TGGGTAAT & TCAGCATIA & AGATCAGTAA & TGTC & ACATAGACCA & GGAAACCCTA & TGCTAG & GC & \\
\hline $512 \mathrm{Abn}-\mathrm{A}$ & TATGCTTGC & ATGGGTAAT & AACAGCATTA & AGATCAGTAA & TGTC & ACATAGACCA & GGGAA & TGCTGGTAAA & GCAA & AGTG \\
\hline Y454176-A3 & TATGCTTGC & ATGGGTAAT & AAACGCATTA & AGATCAGTGC & GGCAI & ACACAGACCA & GGGAATCCTA & TGITAGTGAA & GCAAA & AGTG \\
\hline B1 & ATGCCTCC & ATGGGTAAT & TCAGCATIA & AGGGCCGTAA & TGGC & CCATAAGCCA & GGGAATCCAA & GCTAG & ACAMA & AATG \\
\hline B2 & CTGC & TGGGTAAT & TCAGC & AGAGCTGTAA & GGC & TCATA & GGGAA & GCTAGTAAA & ACAAA & AATGA \\
\hline & TGC & AAT & A & AGAGC & $5 G C$ & & $A A$ & T & & AA \\
\hline & 11 & $\ldots \ldots l$ & $\ldots \ldots$ & & & & & & & 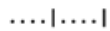 \\
\hline & 1405 & 1415 & 142 & 14 & 14 & 14 & 1465 & 1475 & 14 & 1495 \\
\hline & ATGAAGATTT & ATAGCTAGA & TATTAGAAG & AATTGATGC & GAACCTGTI & CTGAGCCTA & AAAAA & CTAA & $\mathrm{ACTCT}$ & $A C A C$ \\
\hline A2 & IGAAGATIT & TCGCAAGA & ACTA & IGC & TGAG & TA & AAAA & CTTGA & AT & ATA \\
\hline A & TGAAGACTT & TAGCAAGA & IGTTAGAAG & CATAGATGC & SAACCAGTT & CAGAT & AAAAACATA & TTTGAA & ACTCT & $A C A C$ \\
\hline A3 & TGAAGATIT & ATAGCCAGG & TATTAGAAG & TATAGATGC & SAGCCAGTT & CCTA & $A A Q A$ & TITGAAAGTA & ACTCI & ATAC \\
\hline บe10120-B1 & GAGGAATT & CAGCAAGA & GCTAGAAG & ATAGATGC & AAGCCAGTT & CCAGCCCA & AAAGAATA & TCTAAAGGTA & САTTATCTT & ATACAAATGC \\
\hline B2 & GAAGACTT & CAGCAAGA & GCTAGAAG & ATAGATGC & AACCAGTT & ACAACCTA & AGGGAATA & ITAAAATTA & СTCTGTCAT & TACAAATGC \\
\hline & IGGATIT & CAGCAAGG & CTAGAAG & AGATEr & & & & & & \\
\hline
\end{tabular}
\end{abstract}

Fig. 2. Comparison of two field strains from farm E with the reference strain (subtype A1, A2, A3, B1, B2).

be able to detect an infected animal prior to seroconversion, but due to low viral load in the post seroconversion phase of infection, PCR tests are generally less sensitive than ELISA techniques. It is suggested that the combination of serology and PCR together with relatively short sampling intervals is optimal for detecting SRLV infected animals especially in eradication programmes where the early detection of infection is needed (De Andrés et al. 2005).

In the Czech Republic, an eradication program has never been officially established but breeding flocks under official control of production efficiency were systematically tested for presence of MVV/CAEV antibodies since late 1990s. This attempt has reduced the prevalence of SRLVs in these flocks under $1 \%$. However, there is no exact defined number of other flocks out of this program, where the detected serological prevalence of MVV in sheep was $19.9 \%(556 / 2801)$, and of CAE in goats $14.1 \%(86 / 609)$. Eventually, on 7 farms out of 21 (33\%) the SRLV infection was detected. Prevalence within individual herds ranged between 4.5 and 56.2\% (Bartak et al. 2017).

This study represents the first report of genetic characterisation of SRLV strains circulating within the territory of the Czech Republic. Previous study reporting about the phylogenetic analysis of the MVV strains in the Czech Republic (Celer et al. 2000) comparing partial gag gene sequences considered the relevance to prototype MVV isolates K1514, EV1 and SA-OMVV without further recently used identification of the genotype. Our results identified the genotypes in 77 sequences, of which 60 were genotype A present in both animal species, and 17 belonged to genotype B occurring in one sheep flock. Ovine and goat sequences, which belong to genotype A could not be assigned to existing subtypes, however, they were found to be related to subtype A2 or A3. Further analyses have to be performed on larger segments of gag-pol and env or rev gene to classify the relevance to existing subtypes. The length of gag gene part analysed in our study was defined by the size of the reamplification product for the reason of the detection of sensitivity. Published data on the distribution of SRLV genotypes and subtypes in goats and sheep from different countries (Ramírez et al. 2012) state the presence of subtype A2 in Canada, Finland, 
Turkey, and USA, meanwhile subtype A3 was-declared in Spain, Switzerland, and Turkey. In contrast, the genotype B strains limited only to one flock were clearly identified as subtype B2 occurring in France, Italy, Poland, Spain and Switzerland.

Maedi-visna virus prototypes originally isolated from sheep are now referred to as group A, further divided into several subtypes isolated from sheep, goats or both species (Shah et al. 2004a,b). Caprine arthritis-encephalitis virus prototypes originally isolated from goats are referred to as group B, divided into subtypes as well isolated from both species (Pisoni et al. 2005; Shah et al. 2004a). It is clearly confirmed by numerous other studies from several countries that CAEV and VMV often cross the species barrier infecting the new host, persisting in it and spreading across the new host population (Valas et al. 1996; Leroux et al. 1997; Germain et al. 2006). Our study revealed the parallel occurrence of genotype A in a mixed flock on farm A and "CAEV-like" genotype B in sheep on farm E. Thus, even if some genotypes might have been originally assigned to a single host species, the host species spectrum may be wider in nature.

Small ruminant lentivirus displays a high genetic and antigenic heterogeneity and includes diverse genotypes and subgenotypes that display great genetic variability. The molecular epidemiology of SRLV is constantly changing due to animal movements, cross-species transmission and due to their rapid evolutionary rate. Mutations are very frequent in lentiviruses and also the recombination can occur between different SRLVs, creating new variants. Heterogeneous SRLV genotypes may affect the diagnostic sensitivity and specificity. The development of assays tailored by region might help resolve the problem of high mutation rates of SRLV. Sequencing of lentivirus isolates from the area of interest is a prerequisite for this approach. The phylogenetic analysis and nucleotide diversity comparison proved the variability of analysed viral strain across different Czech flocks, except for farm $\mathrm{C}$ which had sequences clustered together with two other farms (Farm A and D). On farm E, where mixed infection genotypes A and B were detected in sheep, there is the possibility of introduction of some of them by the purchase of animals from another country.

Comparing the serological and molecular approaches to determining the SRLV genotypes in positive flocks, the results of ELISA designated for that purpose (Eradikit SRLV ELISA test) coincided in the majority of the tested samples with the molecular characterisation of genotypes. There were some exceptions in several individual samples where the genotype was not correlated in both methods, which can be attributed to lower ELISA specificity. If ELISA is used on the flock level rather than the individual level, it could be a suitable tool to differentiate genotypes $\mathrm{A}$ and $\mathrm{B}$ in case the sequencing analysis is not available in the laboratory.

This study describes an epidemiological situation of SRLV in the Czech Republic. It declares the presence of two SRLV genotypes A and B within the territory of the country and the distribution of different subtypes in sheep and goats. The data reported in this study suggest that Czech SRLV strains are more divergent within the A genotype and further characterization of the A2/A3 strains should be performed to classify them precisely or to confirm a new subtype.

\section{Acknowledgements}

This work was supported by the Ministry of Agriculture of the Czech Republic with the project NAZV QJ1610096.

\section{References}

Alvarez V, Daltabuit-Test M, Arranz J, Leginagoikoa I, Juste RA, Amorena B, de Andres D, Lujan L, Badiola JJ, Berriatua E 2006: PCR detection of colostrumassociated Maedi-Visna virus (MVV) infection and relationship with ELISA-antibody status in lambs. Res Vet Sci 80: 226-34 
Barták P, Václavek P, Kostková M, Mikulášková K, Šimek B 2017: Prevalence of lentiviral diseases in small ruminants in the Czech Republic with the use of serologic diagnostic. Veterinářství 67: 227-232

Blacklaws BA, Berriatua E, Torsteinsdottir S, Watt NJ, de Andres D, Klein D, Harkiss GD 2004: Transmission of small ruminant lentiviruses. Vet Microbiol 101: 199-208

Celer V 1997: Diagnostic use of structural proteins of the Maedi-Visna virus (in Czech). Dissertation, University of Veterinary and Pharmaceutical Sciences Brno

Celer V Jr, Němcová H, Celer V 1997: Isolation and partial characterization of ovine lentivirus in the Czech Republic. Folia Microbiol (Praha) 42: 395-399

Celer V Jr, Celer V, Nejedlá E, Bertoni G, Peterhans E, Zanoni RG 2000: The detection of proviral DNA by seminested polymerase chain reaction and phylogenetic analysis of Czech Maedi-Visna isolates based on gag gene sequences. J Vet Med B Infect Dis Vet Public Health 47: 203-215

De Andrés D, Klein D, Watt NJ, Berriatu A E, Torsteinsdottir S, Blacklaws BA, Harkiss GD 2005: Diagnostic test for small ruminat lentiviruses. Vet Microbiol 107: 49-62

Germain K, Valas S 2006: Distribution and heterogeneity of small ruminant lentivirus envelope subtypes in naturally infected French sheep. Virus Res 120: 156-162

Gifford RJ 2012: Viral evolution in deep time: Lentiviruses and mammals. Trends Genet 28: 89-100

Gjerset B, Jonassen CM, Rimstad E 2007: Natural transmission and comparative analysis of small ruminant lentiviruses in the Norwegian sheep and goat populations. Virus Res 125: 153-156

Grego E, Bertolotti L, Quasso A, Profiti M, Lacerenza D, Muz D, Rosati S 2007:Genetic characterization of small ruminant lentivirus in Italian mixed flocks: evidence for a novel genotype circulating in a local goat population. J Gen Virol 88: 3423-3427

Hall TA 1999: "BioEdit: a user-friendly biological sequence alignment editor and analysis program for Windows 95/98/NT”. Nucl Acids Symp Ser 41: 95-98

Kumar S, Stecher G, and Tamura K 2016: MEGA7: Molecular Evolutionary Genetics Analysis version 7.0 for bigger datasets. Mol Biol Evol 33: 1870-1874

L'Homme Y, Ouardani M, Lévesque V, Bertoni G, Simard C, Pisoni G 2011: Molecular characterization and phylogenetic analysis of small ruminant lentiviruses isolated from Canadian sheep and goats. Virol J 8: 271

Leroux C, Chastang, J, Greenland T, Mornex JF 1997: Genomic heterogeneity of small ruminant lentiviruses: Existence of heterogeneous populations in sheep and of the same lentiviral genotypes in sheep and goats. Arch Virol 142: 1125-1137

Narayan O, Kennedy-Stoskopf S, Sheffer D, Griffin DE, Clements JE 1983: Activation of caprine arthritisencephalitis virus expression during maturation of monocytesto macrophages. Infect Immun 41: 67-73

Narayan O, Clements JE 1989: Biology and pathogenesis of lentiviruses. J Gen Virol 70: 1617-1639

Nei M, Kumar S 2000: Molecular Evolution and Phylogenetics. Oxford University Press, New York

Pepin M, Vitu C, Russo P, Mornex JF, Peterhans E 1998: Maedi-visna virus infection in sheep: a review. Vet Res 29: $341-367$

Pisoni G, Bertoni G, Boettcher P, Ponti W, Moroni P 2005: Phylogenetic analysis of the gag region encoding the matrix protein of small ruminant lentiviruses: comparative analysis and molecular epidemiological applications. Virus Res 116: 159-167

Ramírez H, Reina R, Amorena B, de Andrés D, Martínez HA 2013: Small ruminantlentiviruses: genetic variability, tropism and diagnosis. Viruses 23: 1175-1207

Reina R, Bertolotti L, Dei Giudici S, Puggioni G, Ponti N, Profiti M, Patta C, Rosati S 2010: Small ruminant lentivirus genotype $\mathrm{E}$ is widespread in Sarda goat. Vet Microbiol 144: 24-31

Shah C, Boni J, Huder JB, Vogt HR, Muhlherr J, Zanoni R, Miserez R, Lutz H, Schupbach J 2004a: Phylogenetic analysis and reclassification of caprine and ovine lentiviruses based on 104 new isolates: Evidence for regular sheep-to-goat transmission and worldwide propagation through livestock trade. Virology 319: 12-26

Shah C, Huder JB, Böni J, Schönmann M, Mühlherr J, Lutz H, Schüpbach J 2004b: Direct evidence for natural transmission of small-ruminant lentiviruses of subtype A4 from goats to sheep and vice versa. J Virol 78 : 7518-7522

Valas S, Benoit C, Guionaud C, Perrin G, Mamoun RZ 1997: North American and French caprine arthritisencephalitis viruses emerge from ovine maedi-visna viruses. Virology 237: 307-318 